\title{
DESENVOLVIMENTO DE APLICATIVO PARA ENSINO DE DINÂMICA DOS FLUIDOS COMPUTACIONAL
}

Eduardo Henrique Taube Cunegatto - eduardohtcunegatto@aluno.santoangelo.uri.br Universidade Regional Integrada do Alto Uruguai e Missões - Campus de Santo Ângelo Av. Universidade das Missões, 464

98802-470 - Santo Ângelo - Rio Grande do Sul

Cleiton Elsner da Fonseca - cfonseca@san.uri.br

Universidade Regional Integrada do Alto Uruguai e Missões - Campus de Santo Ângelo Av. Universidade das Missões, 464

98802-470 - Santo Ângelo - Rio Grande do Sul

Flávio Kieckow-fkieckow@san.uri.br

Universidade Regional Integrada do Alto Uruguai e Missões - Campus de Santo Ângelo Av. Universidade das Missões, 464

98802-470 - Santo Ângelo - Rio Grande do Sul

Flavia Schwarz Franceschini Zinani-fzinani@unisinos.br

Universidade do Vale do Rio dos Sinos

Av. Unisinos, 950

93022-750 - São Leopoldo - Rio Grande do Sul

Resumo: A presente pesquisa tem por objetivo apresentar o desenvolvimento de um aplicativo educacional, com o intuito de fornecer um suporte didático ativo para o ensino de Introdução à Dinâmica dos Fluidos Computacional, o SimuLearn 2D. A ideia principal é possibilitar uma maneira interativa para o aprendizado com o uso do aplicativo. O programa busca apresentar uma seção de lições interativas de simulação e pode ser usado para fins de estudo e pesquisa. $O$ solver a ser utilizado é baseado na solução das equações de balanço de quantidade de movimento linear, da equação da continuidade e do balanço de energia, aplicando o método numérico de volumes finitos. O código foi desenvolvido na linguagem FORTRAN 90, enquanto a interface do software foi desenvolvida em PYTHON. No presente trabalho, são apresentados exemplos de simulações de problemas exemplo utilizando o SimuLearn 2D, mostrando análises de variações de parâmetros e de comparações com resultados da literatura, a fim de demonstrar a validação e o desempenho do solver desenvolvido.

Palavras-chave: Software educativo. Dinâmica dos Fluidos Computacional

\section{INTRODUÇÃO}

A ascensão da internet e a facilidade do acesso a computadores proporcionam numerosas maneiras de promover o ensino nas mais diversas áreas. Temáticas, que outrora eram de difícil aplicabilidade, principalmente no quesito prático, tornaram-se totalmente acessíveis por meio do uso de aplicativos. Aplicativos voltados à educação oferecem o compartilhamento de conhecimento de maneira dinâmica e interativa, tornando aprendizado instigante e agradável, mesmo em áreas de grande dificuldade de entendimento. Observando essas características, a utilização de aplicativos de Dinâmica dos Fluidos, uma das áreas mais importantes para 
desenvolvimento da ciência e engenharia, pode ser uma ferramenta útil para a construção do conhecimento de forma simples e didática.

A ciência da computação viabilizou o uso da tecnologia na educação, utilizando o computador como ferramenta de aprendizagem. O Pensamento Computacional (PC) é uma dessas estratégias que vem ganhando força nesta última década como mostra a pesquisa de Zanete, Borges e Ricarte (2016). Os pesquisadores Brennan e Resnick (2012) mostraram que uma abordagem baseada em PC pode ser utilizada no aprendizado de programação, principalmente para jovens, usando ferramentas que motivem e estimulem a criatividade. O PC é uma ótima estratégia para modelar soluções e resolver problemas de forma eficiente.

Processos e tecnologias descritos pela Dinâmica dos Fluidos possuem complexidade física e matemática, exigindo modelagem através de equações diferenciais parciais, geralmente sem solução analítica. Para a solução dessas modelagens, se faz necessário o desenvolvimento de ferramentas numéricas como as utilizadas na Dinâmica dos Fluidos Computacional (Computational Fluid Dynamics - CFD), a qual o objetivo é prever o comportamento de problemas de fenômenos de transporte reais através de métodos numéricos. Dentre os métodos numéricos aplicados a CFD podemos destacar o Método de Volumes Finitos (MVF) (PATANKAR, 1980) devido à natureza de sua formulação, a qual tem como princípio integrar as equações de balanço governantes em volumes finitos discretos no domínio computacional, garantindo a conservação das propriedades.

Atualmente, estão disponíveis inúmeros softwares de CFD para utilização em engenharia. Entretanto, não são comuns os softwares de CFD voltados ao ensino. Os alunos em nível de graduação geralmente encontram dificuldades em utilizar os softwares comerciais e livres, pois os mesmos são voltados à aplicação, e não ao entendimento dos métodos e dos problemas.

Diante dos argumentos apresentados, o presente artigo expõe a implementação de uma metodologia didática ativa na forma de um aplicativo educacional que auxilia no aprendizado de Dinâmica dos Fluidos, de forma que os conceitos sejam absorvidos de forma interativa e que seja possível obter soluções de simulações computacionais de problemas básicos de engenharia, além de facilitar o entendimento físico dos problemas bem como a sua visualização. Para a criação do solver, foram utilizadas as equações da conservação de massa, a equação da quantidade de movimento dos fluidos e a equação da energia, sendo elas solucionadas pelo MVF.

\section{METODOLOGIA}

O aplicativo busca ensinar ao usuário, por meio de uma metodologia de ensino ativa, a maneira na qual equações governantes de Dinâmicas dos Fluidos podem ser utilizadas para criação de modelos computacionais que simulam os fenômenos reais. Ao mesmo tempo, o aplicativo pretende ensinar a metodologia para a realização das simulações.

\subsection{Método didático}

Busca-se implementar no aplicativo uma metodologia ativa, na qual o usuário possa aprender com o programa, de modo que o mesmo seja o protagonista, fazendo com que seu conhecimento seja absorvido de forma autônoma e, ao mesmo tempo, participativa, por meio da interação com o aplicativo. Dentro do escopo de metodologia ativa, existem diversas ramificações nas quais podem introduzir diferentes técnicas de ensino. A que mais se encaixa nos objetivos do aplicativo é a prática da Aprendizagem Baseada em Problemas, que tem como propósito tornar o usuário capaz de construir seu aprendizado conceitual, procedimental e 
atitudinal através de problemas propostos, expondo a situações motivadoras e o prepara para o entendimento do conteúdo (BOROCHOVICIUS, 2014).

\subsection{Modelagem matemática}

A modelagem matemática empregada nas simulações numéricas dos problemas aplicados compõe-se da equação da continuidade, a equação de balanço de quantidade de movimento para um fluido Newtoniano generalizado, e a equação de balanço de energia em termos da temperatura dadas respectivamente por:

$$
\begin{gathered}
\nabla \cdot \boldsymbol{u}=0 \\
\nabla \cdot(\rho \boldsymbol{u u})=-\nabla p+\mu \nabla \cdot \boldsymbol{D}=\boldsymbol{f} \\
\nabla \cdot(\boldsymbol{u} T)=\nabla \cdot(\alpha \nabla T)+S
\end{gathered}
$$

onde $\boldsymbol{u}$ é o campo de velocidades, $\rho$ é a massa específica, $p$ é a pressão estática, $\boldsymbol{f}$ é o tensor de forças de corpo, $T$ é o campo de temperaturas, $\alpha$ é a difusividade térmica, $S$ é a fonte de energia térmica e $\mu$ é a viscosidade. O tensor taxa de deformação, $\boldsymbol{D}$, é dado por:

$$
\boldsymbol{D}=\nabla \boldsymbol{u}+\nabla \boldsymbol{u}^{T}
$$

As equações (1)-(4) são adimensionalizadas por meio das variáveis e números adimensionais definidos pela Equação (5):

$$
R_{e}=\frac{\rho V_{c} L_{c}}{\mu} ; x^{*}=\frac{x}{L_{c}} ; y^{*}=\frac{y}{L_{c}} ; T^{*}=\frac{T}{T_{r}}
$$

onde $V c$ é a velocidade característica, $L c$ é o comprimento característico, $\operatorname{Tr}$ é a temperatura de referência, $R e$ é o número adimensional de Reynolds e os subscritos * definem as variáveis adimensionais.

\subsection{Modelagem numérica}

Para a solução das equações descritas pela modelagem matemática, Equações (1)-(3), foi utilizado o método dos volumes finitos. Optou-se pelo seu uso pois sua formulação tem por característica, para obtenção das equações aproximadas, satisfazer a conservação das propriedades em nível de volumes elementares (MALISKA, 2004).

Em relação à discretização espacial dos termos advectivos, foram utilizados os métodos Upwind de primeira ordem e Power Law. Já para o acoplamento pressão-velocidade, fez-se uso da formulação SIMPLE (Semi Implicit Linked Equations). Seu uso permite facilidade de implementação, devido à forma explícita na qual as equações são interligadas (PATANKAR, 1980).

\subsection{Estrutura computacional}

O aplicativo foi desenvolvido em duas linguagens computacionais: para a interface gráfica, utiliza-se PYTHON e para o solver, FORTRAN 90. A interface gráfica é a parte predominante do aplicativo permitindo ao usuário o acesso às funcionalidades do programa e bem como a entrada de dados para as simulações. Também por meio dela que a abordagem didática é feita. Os dados são transmitidos para o solver através de um arquivo. Esses dados são lidos pelo 
solver, o qual os utiliza para solução das equações. Os resultados são impressos em arquivos e também são disponíveis para visualização na seção de pós-processamento.

\section{Interface}

A escolha da linguagem PYTHON para criação da interface está no fato dela ser uma linguagem eficiente, isto é, poucas linhas de código são necessárias, gerando assim um código mais "limpo" e de fácil compreensão (MATTHES, 2016). Além disso, a linguagem conta com diversas bibliotecas, como a wXPython, que oferece diversas ferramentas para criação de interfaces gráficas, sendo esta a utilizada no projeto. Também é utilizada a biblioteca Matplotlib, a qual proporciona inúmeras maneiras de visualização de dados, sendo ideal para desenvolvimento do pós-processamento dos resultados simulados no aplicativo.

\section{Solver}

O desenvolvimento do solver foi feito utilizando a linguagem computacional FORTRAN 90, que em sua essência, é apropriado para uso científico. Essa linguagem permite criação de programas com alta velocidade de execução, o que justifica a escolha neste projeto.

\section{RESULTADOS}

Nesta seção, apresenta-se a fase atual do desenvolvimento do aplicativo denominado SimuLearn 2D, demonstrando a forma de iteração aplicativo-usuário e aplicações da ferramenta em benchmarks de Dinâmica dos Fluidos.

\subsection{Interface interativa}

Iniciando o aplicativo, o usuário vai se deparar com a tela de menu principal onde todos os recursos da ferramenta podem ser acessados, sendo observado na Figura 1(a). Ao clicar no botão Theory, o usuário é direcionado à seção destinada à documentação da teoria utilizada no aplicativo, onde são apresentados todos os conceitos relacionados à Dinâmica dos Fluidos, Transferência de Calor e métodos numéricos utilizados, incluindo uma seção de bibliografias recomendadas, permitindo o usuário escolher um assunto específico no qual tem interesse por meios dos botões laterais, como mostra a Figura 1(b). A parte interativa do aplicativo também é acessada pelo menu principal através da seção Lesson. Esta tem por objetivo introduzir o usuário aos principais conceitos de simulação numérica e conceitos físicos de mecânica dos fluidos e transferência de calor.

Como metodologia de aplicação didática, a seção Lesson apresenta uma estrutura sistemática onde é introduzido os conceitos de cada etapa da lição, seguido de uma interação entre a própria lição e o usuário e finalmente uma avaliação dos conhecimentos em forma de desafios variados como quizzes, games e testes.

A Figura 2 mostra o fluxograma da seção Lesson. Inicialmente, é mostrada uma introdução sobre CFD, com palavras-chave destacadas. O usuário pode interagir com essas palavras-chave clicando sobre elas, escolhendo um tema específico para maiores informações. Após a introdução, o programa dá ao usuário algumas opções de benchmarks serão utilizados na lição. Ao selecionar a opção de benchmark, é mostrada uma explicação sobre o mesmo, para que o usuário possa entender a física do problema. Na sequência da lição, o usuário define o domínio de sua simulação, utilizando seus próprios dados. O usuário também define a geração da malha, as condições de contorno e as configurações dos cálculos, com base nas explicações fornecidas pelo programa. Para avaliar o conhecimento adquirido, em cada etapa, um desafio é lançado e só será possível avançar se o usuário atingir o critério de avaliação exigido. 


\section{COBENGE de Educação em Engenharia de Educaçāo em Engenharia

Figura 1 - Menus do aplicativo SimuLearn 2D: (a) Menu principal; (b) Theory
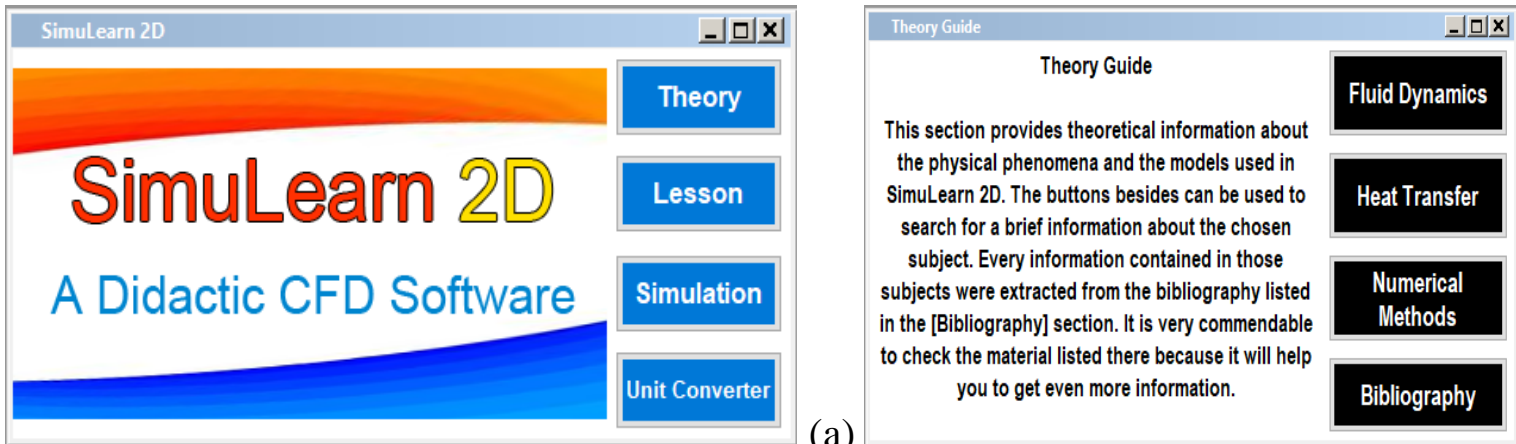

Fonte: Autores (2020).

A lição continua com a interpretação dos resultados após o pós-processamento, onde são apresentados os campos de velocidade, pressão e temperatura e perfis das variáveis. Nessa etapa também são demonstrados possíveis erros gerados nas simulações, dando condições ao usuário de identificar e corrigir os problemas.

Figura 2 - Fluxograma da seção Lesson do programa.

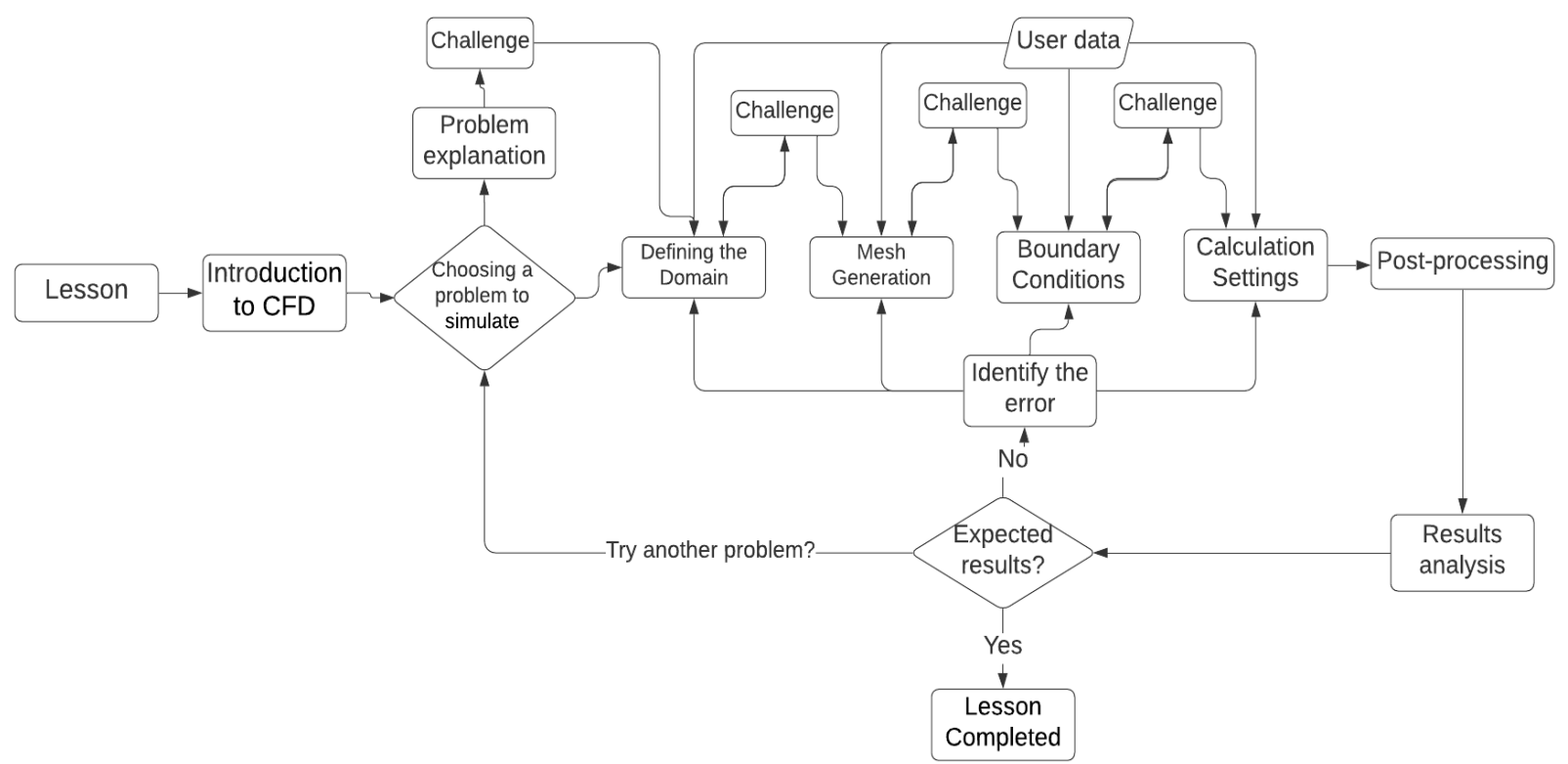

Fonte: Autores (2020).

\subsection{Aplicação de simulações}

Afim de apresentar a aplicabilidade do solver, realizou-se simulações de benchmarks de Dinâmica dos Fluidos. Os problemas escolhidos foram o escoamento entre placas planas e escoamento sobre uma cavidade, demonstradas na Figura 3.

A Figura 3(a) mostra o escoamento entre duas placas planas onde o objetivo dela é demonstrar o escoamento de um fluido em um duto. Para o domínio da simulação, utilizou-se critérios adimensionais, com relação $L x / H y=20$. As condições de contorno são de entrada de velocidade prescrita (inlet) com perfil flat, condição de não deslizamento nas paredes e condição de saída de fluxo livre (outlet). 
Figura 3 - Desenhos esquemáticos das simulações: (a) Escoamento entre placas planas; (b) escoamento sobre uma cavidade.

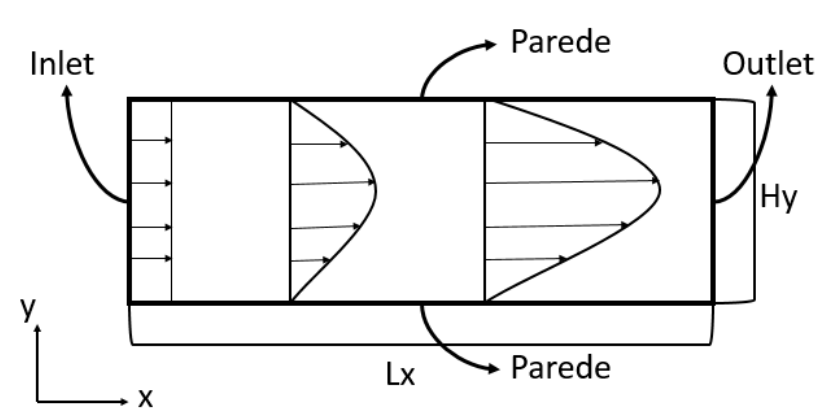

(a)

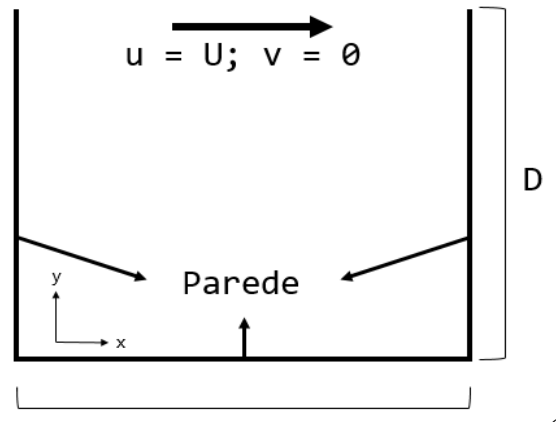

Fonte: Autores (2020).

Em relação à Figura 3(b), representa o escoamento em uma geometria que representa uma cavidade. O objetivo é analisar o comportamento do escoamento diante de tal situação. Utilizou-se razão de aspecto igual a 1, condições de não deslizamento nas paredes e velocidade prescrita. Para ambas simulações, realizou-se um teste de convergência de malha (GCI - Grid Convergence Index) utilizando como critério de variável principal a velocidade, variando em $30 \%$ (ASME, 2008) o refinamento da malha e critério de erro entre as variáveis em $1 \%$.

A Figura 4 mostra os resultados da simulação do problema do escoamento entre placas planas para $R e=10$. Os resultados apresentados mostram coerência com os dados vistos na literatura. É possível observar que o programa foi capaz de prever o desenvolvimento do escoamento, Figura 4(a), apresentando um perfil desenvolvido ao longo do duto. A Figura 4(b) mostra o campo de velocidades da componente $y$, onde é possível observar o fenômeno de entry flow, na entrada, onde há mudanças no campo de velocidades, indicando que após tal fenômeno, o perfil de velocidades encontra-se desenvolvido. Já a Figura 4(c) é apresentado o campo de pressão, onde é possível observar o decaimento da mesma, indicando movimentação do fluido, mostrando um perfil paralelo característico, com mudanças apenas na entrada do duto, como era esperado.

Figura 4 - Resultados da simulação de escoamento entre placas planas: (a) Campo de velocidade x; (b) Campo de velocidade y; (c) Campo de pressão.

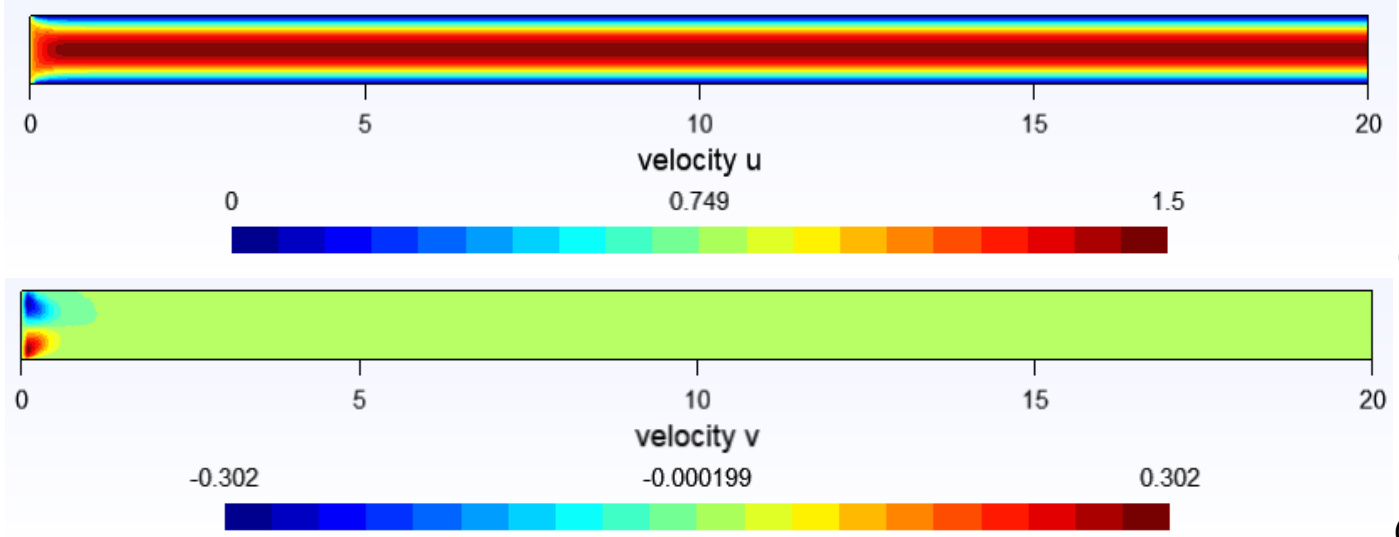

(a) 


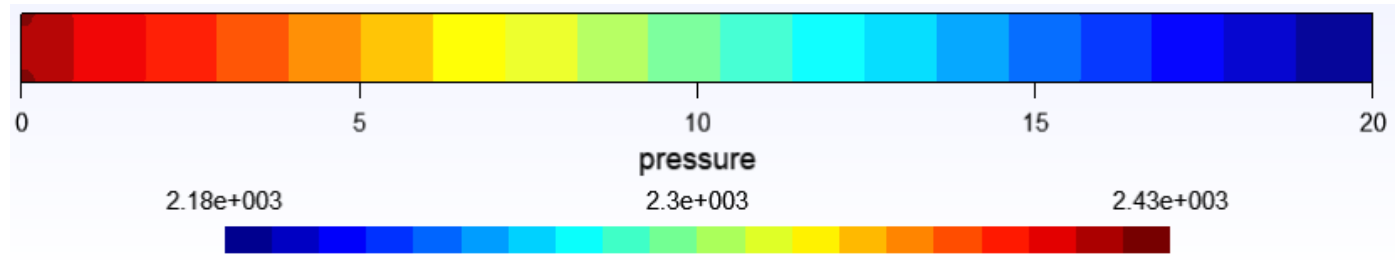

(c)

Fonte: Autores (2020)

Na Figura 5 demonstra-se o perfil de velocidades na direção $x *$ para $R e=10$, onde é possível observar os efeitos da condição de não-deslizamento sobre o escoamento, indicando que quando em contato com a parede, a velocidade é nula, ao passo que, afastando-se da superfície sólida, aumenta-se gradativamente, até que, no centro do domínio, atinge seu valor máximo, formando o perfil desenvolvido. Com o teste de convergência de malha, demonstrou-se que o solver reproduziu resultados consistentes e idênticos à solução exata do problema, até mesmo para as diferentes configurações de malha, indicando a capacidade de prever o fenômeno físico do problema.

Figura 5 - Resultados do escoamento entre placas planas: Perfil de velocidades $\mathrm{X}$

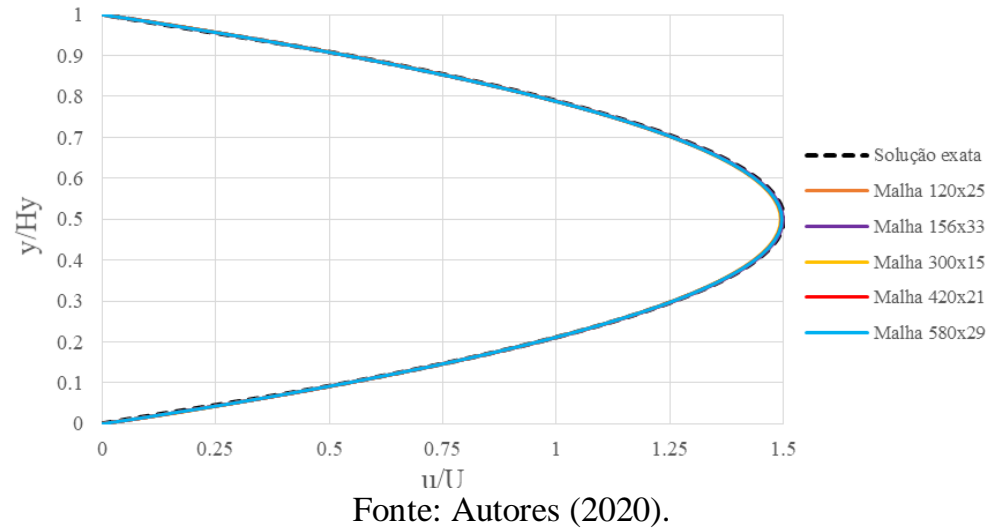

Na Figura 6 estão os resultados obtidos na simulação do escoamento em cavidade. São ilustrados os campos de velocidade da componente $x$ para $R e=100$ e $R e=400$, respectivamente.

Os resultados mostram que a componente da velocidade $x$ tem um decaimento por conta da presença das paredes, caracterizada por uma região onde as velocidades mudam de direção. Essa região tende a se deslocar para o sul com o aumento do número de Reynolds devido ao escoamento apresentar uma maior quantidade de movimento fazendo com que ele fique permaneça colado na parede em uma área maior.

Além dos campos de velocidade, também foram retirados resultados que mostram o comportamento das linhas de corrente do problema, sendo estes demonstrados pela Figura 6 (c) e (d). Isso permite observar o fenômeno característico do problema, que se trata da presença de vórtices. Como mencionado, o aumento da quantidade de movimento também faz com que o olho do vórtice mude de posição, além de, no canto inferior direito, ocasionar a formação de novos vórtices. 
Figura 6 - Campos de velocidade $u$ e linhas de corrente do escoamento sobre cavidade: (a) e (c) $\operatorname{Re}=100$; (b) e (d) $\operatorname{Re}=400$.
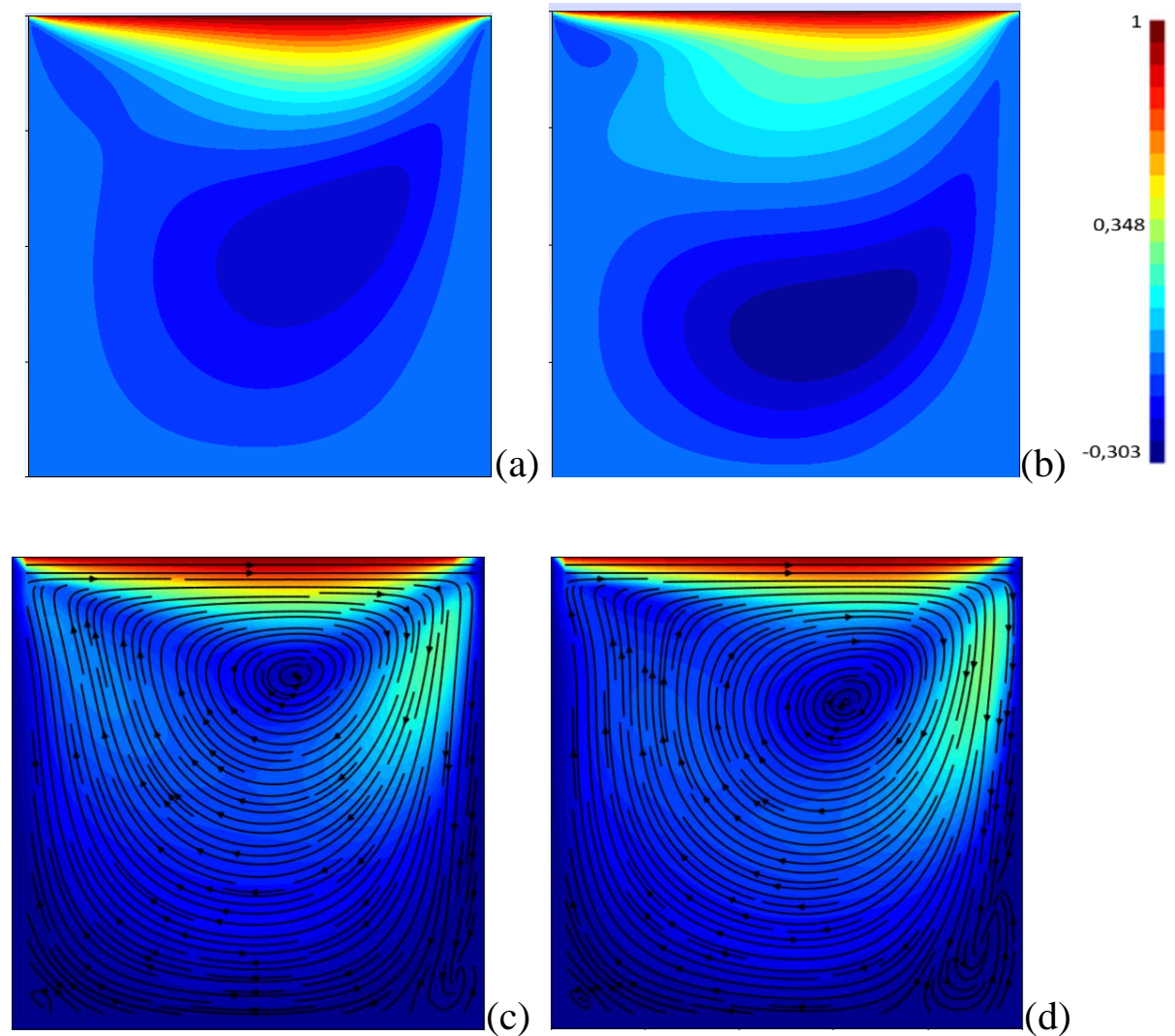

(d)

Fonte: Autores (2020).

Foi feita uma comparação dos resultados, onde utilizou-se como critérios de referência dados da literatura de (GHIA. et al. 1982) e (ABDELMIGID. Et al. 2016) e simulações no software comercial ANSYS Fluent. O resultado desta comparação é apresentado por meio do gráfico do perfil de velocidades do problema na posição y/D = 0.5, mostrado na Figura 7.

Figura 7 - Perfis de velocidade $x$ da simulação do escoamento sobre cavidade: (a) Re=100; (b) $\operatorname{Re}=400$.
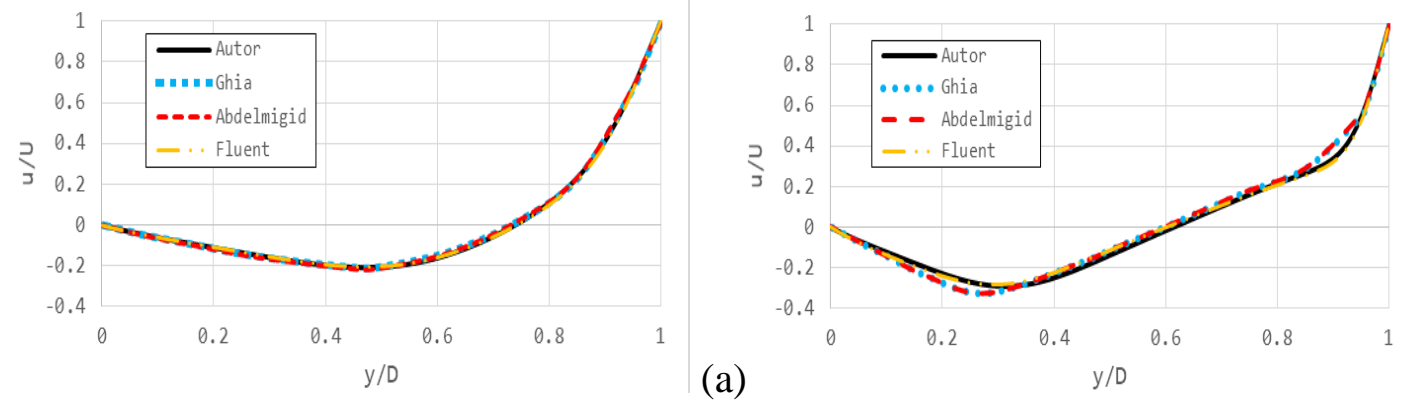

(b)

Fonte: Autores (2020).

O comportamento dos perfis mostrado no gráfico da Figura 8(a) são idênticos comparados aos resultados literários e simulados, mostrando grande precisão do solver. Já para $\operatorname{Re}=400$, os resultados obtidos no programa estão muito próximos em relação aos do software Fluent. 
Entretanto, em relação ao perfil dos pesquisadores, existe uma pequena diferença, mas o comportamento do perfil entre os demais ainda é similar.

\section{CONSIDERAÇÕES FINAIS}

A Dinâmica dos Fluidos Computacional é uma área em acelerada expansão e em constante desenvolvimento. No entanto, percebe-se dificuldade por parte dos estudantes compreender os princípios dessa ferramenta e por parte dos professores em transmitir o conhecimento, utilizando as ferramentas comerciais com foco em aplicações em engenharia. Assim, o presente trabalho propõe o desenvolvimento de uma ferramenta educativa de CFD e apresenta seu desenvolvimento e resultados iniciais.

No que concerne à metodologia didática adotada neste trabalho, buscou-se implementar uma metodologia atual, o Pensamento Computacional, sendo esta uma tendência nos meios de ensino, onde o aplicativo poderá ser utilizado em meios acadêmicos e científicos ou até mesmo no treinamento de profissionais que buscam especialização na área.

A próxima etapa do projeto é avaliar o desempenho do SimuLearn $2 D$ através da aplicação com alunos de graduação e pós-graduação. Esta pesquisa visará ainda a melhoria do aplicativo.

Com relação à metodologia numérica, confirmou-se que os conceitos aplicados foram extremamente eficazes, permitindo a geração de um solver funcional e proporcionou uma visão profunda sobre o funcionamento de simuladores de fenômenos estudados, e consequentemente, a análise da veracidade dos resultados simulados com o fenômeno real.

Por fim, os resultados obtidos no solver atenderam às expectativas, mostrando-se adequados aos obtidos na literatura, demonstrando que o código está pronto a ser aplicado para realizar simulações em dinâmica dos fluidos, tanto em um contexto didático, quanto científico.

\section{REFERÊNCIAS}

ABDELMIGID, Tamer. et al. Revisiting the lid-driven cavity flow problem: Review and new steady state benchmarking results using GPU accelerated code. AEJ - Alexandria Engineering Journal, 2016.

AMERICAN SOCIETY OF MECHANICAL ENGINEERS. Procedure for Estimation and Reporting of Uncertainty Due to Discretizationin CFD Applications. Journal of Fluids Engineering, v. 130, 2008.

BOROCHOVICIUS, Eli; TORTELLA, Jussara Cristina Barboza. Aprendizagem Baseada em Problemas: um método de ensino-aprendizagem e suas práticas educativas. Ensaio: aval.pol.públ.Educ. Rio de Janeiro, v. 22, n. 83, p. 263-294, 2014.

BRENNAN, K; E RESNICK, M. New frameworks for studying and assessing the development of computational thinking. In Proceedings of the 2012 annual meeting of the American Educational Research Association, Vancouver, Canada, 2012.

GHIA, U.; GHIA, K.N.; SHIN, C.T. High-Re solutions for incompressible flow using the Navier-Stokes equations and a multigrid method. Journal of Computational Physics, 1982.

MALISKA, Clovis R. Transferência de Calor e Mecânica dos Fluidos Computacional. 2.ed. Rio de Janeiro: LTC, 2004. 
MATTHES, Eric. Curso Intensivo de Python.1.ed. São Paulo: Novatec Editora Ltda, 2016.

PATANKAR, Suhas V. Numerical Heat Transfer and Fluid Flow. 1.ed. Estados Unidos da América: Hemisphere Publishing Corporation, 1980.

ZANETE, H.A.P.; BORGES, M.A.F.; RICARTE, I.L.M. Pensamento Computacional no Ensino de Programação: Uma Revisão Sistemática da Literatura Brasileira. V

Congresso Brasileiro de Informática na Educação (CBIE 2016). Anais do XXVII Simpósio Brasileiro de Informática na Educação (SBIE 2016)

\title{
SOFTWARE DEVELOPMENT FOR COMPUTATIONAL FLUID DYNAMICS TEACHING
}

\begin{abstract}
This research aims to present the development of an educational application, in order to provide an active didactic support for the teaching of Introduction to Computational Fluid Dynamics, the SimuLearn 2D. The main idea is to enable an interactive way for learning with the use of the application. The program seeks to present an interactive simulation lesson section and can be used for study and research purposes. The solver has been based on the solution of the linear motion quantity balance equations, the continuity equation and the energy balance equation, applying the finite volume numerical method. The code was developed in the FORTRAN 90 language, while the software interface was developed in PYTHON. In the present work, examples of simulations of example problems using SimuLearn $2 D$ are shown, showing analysis of parameter variations and comparisons with results from the literature, in order to demonstrate the validation and performance of the developed solver.
\end{abstract}

Keywords: Teaching software, computational fluid dynamics 